\title{
NORMAS SUPREMAS
}

\section{La concepción estándar}

egún una opinión muy difundida, los ordenamientos jurídicos son sistemas normativos:
(a) dinámicos,
(b) provistos de una estructura jerárquica,
(c) en cuyo vértice figura una norma «última» y «suprema».

Esta opinión no persuade.

En primer lugar, es empíricamente falso que los ordenamientos jurídicos modernos sean sistemas normativos puramente dinámicos.

En segundo lugar, la noción de estructura jerárquica no puede ser tratada como una noción unívoca y no problemática, ya que en los ordenamientos jurídicos evolucionados se entrelazan una multiplicidad de relaciones jerárquicas entre normas.

En tercer lugar, no es en absoluto pacífico que en el vértice de cada ordenamiento figure una sola norma (y no, en cambio, una multiplicidad de normas), y, de todos modos, la norma suprema es una variable que depende del tipo de relaciones jerárquicas a las que se hace referencia.

\section{Criterios materiales de validez y de invalidez}

Es dinámico todo ordenamiento en el que los criterios de existencia y validez de las normas son puramente «formales».

Es formal todo criterio que prescinda del contenido de las normas, haciendo exclusiva referencia al modo de producción de aqué- 
llas (esto es, a grandes rasgos, a la identidad del sujeto que las produce y eventualmente al procedimiento de producción) ${ }^{1}$. Si esto es así, ningún ordenamiento moderno es puramente dinámico, en este sentido, al menos por dos razones:

(1) En primer lugar, todos los ordenamientos jurídicos modernos incluyen un criterio de invalidez que hace referencia al contenido de las normas ${ }^{2}$.

En virtud de ese criterio son normas inválidas no sólo aquellas producidas en un modo distinto al establecido por las meta-normas que regulan la producción de normas, sino también aquellas que, a pesar de haber sido producidas «correctamente» desde el punto de vista formal, son incompatibles con normas jerárquicamente superiores. Por ejemplo, en los ordenamientos con una constitución rígida, es inválida toda ley que sea incompatible con la constitución; en casi todos los ordenamientos occidentales modernos, por otra parte, es inválido todo reglamento incompatible con la ley.

Es más, en realidad, algunas normas deben ser no sólo compatibles con las normas superiores, sino incluso deducibles de una norma superior, bajo pena de invalidez ${ }^{3}$. Por ejemplo, en todo Estado de derecho, es inválida toda decisión jurisdiccional que no sea deducible de una norma legislativa preexistente: «En todo delito debe hacerse por el juez un silogismo perfecto. Pondrase como mayor la ley general, por menor la acción conforme o no con la ley, de que se inferirá por consecuencia la libertad o la pena» ${ }^{4}$.

(2) En segundo lugar, todos los ordenamientos jurídicos modernos incluyen un criterio de validez que hace referencia al contenido de las normas.

En virtud de ese criterio, son normas válidas del ordenamiento no sólo las normas «explícitas»-es decir, las normas expresamente for-

${ }^{1}$ H. Kelsen, Introduction to the Problems of Legal Theory (1934), trad. a cargo de B. Litschewsky y S. L. Paulson, Clarendon Press, Oxford, 1992; 56; Id., General Theory of Law and State, Harvard U. O., Cambridge (Mass.), 1945, págs. 112 s.; Id., Théorie pure du droit (1960), trad. Ch. Eisenmann, Dalloz, París, 1962, págs. 258 y ss. Cfr. L. Gianformaggio (ed.) Sistemi normativi statici e dinamici. Analisi di una tipologia kelseniana, Giappichelli, Torino, 1991.

${ }^{2}$ R. Guastini, «Invalidity», Ratio Juris, 7, 1994, págs. 212 y ss.; Id., Il giudice e la legge. Lezioni di diritto constituzionale, Giappichelli, Torino, 1995, cap. 5.

${ }^{3}$ E. Bulygin, Norme, validità, sistemi normativi, Giappichelli, Torino, 1995, págs. 1 y ss.

${ }^{4}$ C. Beccaria, Dei delitti e delle pene (1764), § IV. N. de T.: para la traducción de la cita de Beccaria se ha seguido la versión española : C. Beccaria, De los delitos y de las penas, Alianza editorial (Trad. a cargo de J. A. de las Casas), Madrid, 1990, cap. 4. ${ }^{\circ}$, pág. 31. 
muladas y promulgadas por (o emanadas de) una autoridad normativa competente según el procedimiento adecuado- sino también todas aquellas que están «implícitas» en las primeras $^{5}$.

¿En qué sentido se habla de «normas implícitas»? En este contexto, el vocablo «implícito» debe ser entendido en un sentido bastante amplio, de modo que incluya al menos tres clases de normas:

(a) las normas que pueden ser inferidas a partir de las normas explícitas según esquemas de razonamiento lógicamente válidos y sin la adición de ulteriores premisas (esto es, sin el empleo de premisas que no sean normas explícitas);

(b) las normas que pueden ser válidamente inferidas de las normas explícitas únicamente con la adición de premisas ulteriores; esto es, de premisas que no constituyan a su vez normas explícitas (por ejemplo, definiciones doctrinales de términos usados en la formulación de normas explícitas);

(c) las normas que pueden ser persuasivamente inferidas a partir de las normas explícitas según esquemas de razonamiento no deductivos y lógicamente inválidos (por ejemplo, entimemas, conjeturas sobre la ratio legis, argumentos analógicos, etc.).

Es en virtud de este criterio que todos los ordenamientos jurídicos están llenos de normas «implícitas», no expresadas, elaboradas por los juristas y por los órganos encargados de la aplicación del derecho, a pesar de que la doctrina y la jurisprudencia no son fuentes «formales» del derecho. Desde este punto de vista, la caracterización de los ordenamientos jurídicos como sistemas esencialmente dinámicos parece descuidar la dimensión lato sensu normativa -productora de normas- que es propia de la dogmática y (lo que importa más) de la jurisprudencia.

\section{Variedad de jerarquías normativas}

La noción de estructura jerárquica no es unívoca. En todos los ordenamientos jurídicos evolucionados las normas están ligadas entre ellas por múltiples vínculos de «jerarquía» ${ }^{6}$. En mi opinión, se dan no menos de cuatro posibles tipos de jerarquías normativas:

${ }^{5}$ R. Guastini, «Produzione di norme a mezzo di norme. Un contributo all'analisis del ragionamiento giuridico», en L. Gianformaggio, E. Lecaldano (eds.), Etica e diritto. Le vie della giustificazione razionale, Laterza, Bari, 1986, págs. 173-201.

${ }^{6}$ A. Pizzorusso, Delle fonti del diritto, Comentario del código civil editado por A. Scialoja y G. Branca, Zanichelli, Bologna, 1977, págs. 7 y ss., 103 y ss.; Id., Lezioni di diritto constituzionale, III ed., II Foro Italiano, Roma, 1984, págs. 452 y ss.; 
(1) Jerarquías estructurales o formales: son aquellas que se dan entre las normas producidas por dos poderes normativos distintos cuando uno de los poderes en cuestión debe su fuente de legitimidad al otro. Por ejemplo: incluso en un régimen de constitución flexible, las normas constitucionales y las normas legislativas; las leyes de delegación y los decretos legislativos.

(2) Jerarquías materiales: son aquellas que se dan entre dos normas cuando una de ellas es inválida en caso de que entre en conflicto con la otra. Por ejemplo: en un régimen de constitución rígida, las normas constitucionales y las normas legislativas ordinarias; las leyes y los reglamentos.

Normalmente, toda jerarquía material refleja una jerarquía formal, pero no toda jerarquía formal se resuelve en una jerarquía mate rial. Por ejemplo: en un régimen de constitución flexible las normas de rango legislativo están formalmente, pero no materialmente, subordinadas a las normas constitucionales.

(3) Jerarquías lógicas: son aquellas que se dan entre dos normas cuando una versa (meta-lingüísticamente) sobre la otra (ejemplo: normas sancionadoras y normas de conducta; normas derogatorias y normas por ellas derogadas; definiciones y normas que contienen el término definido).

Si se usa una noción de meta-norma suficientemente amplia para incluir las normas que versan (no propiamente sobre normas sino) sobre actos normativos ${ }^{7}$, entonces toda jerarquía formal es también una jerarquía lógica. En cambio, es cierto que no todas las jerarquías lógicas son también jerarquías formales: no hay ninguna jerarquía formal, por ejemplo, entre normas derogatorias y normas derogadas.

Id., «Cultura e politica nella produzione ed aplicazione del diritto», Quaderni constituzionali, 10, 1990, págs. 77 y ss.; Id., «Fonti (sistema constituzionale delle)», Digesto delle discipline pubblicistiche, VI, UTET, Torino, 1991; G. Tarello, «Gerarchie normative e interpretazione dei documenti normativi», Politica del diritto, 8, 1977, págs. 499 y ss.; Id., L'Interpretazione della legge, Giuffrè, Milano, 1980, 307 y ss.; R. Guastini, «L'illegittimità delle disposizioni e delle norme», Analisi e diritto. Richerche di giurisprudenza analitica, 3, 1992, págs. 175 y ss.; Id., Quindici lezioni di diritto costituzionale, II ed., Giappichelli, Torino, 1992, págs. 136 y ss.; Id., Le fonti del diritto e l'interpretazione, Tratado de derecho privado editado por G. Iudica y P. Zatti, Giuffrè, Milano, 1993, cap. IV.

${ }^{7}$ Cfr. T. Mazzarese, Logica deontica e linguaggio giuridico, CEDAM, Padova, 1989, págs. 19 y ss.; R. Guastini, «Intorno all'uso di "norma” nel linguaggio giuridico», in Nuova civiltà delle macchine, 3, 1985, 47-54; Id., Lezioni sul linguaggio giuridico, Giappichelli, Torino, 1985, cap. I. 
(4) Jerarquías axiológicas: son aquellas que se dan entre dos normas cuando una de ellas tiene valor de «principio» y la otra tiene valor de «simple norma». Por ejemplo: principios fundamentales de una materia dada y sus correspondientes normas de detalle . $^{8}$.

A veces las jerarquías axiológicas se reflejan en otras tantas jerarquías materiales (esto sucede, por ejemplo, cuando los principios de los que se habla están contenidos en una constitución rígida). Pero no siempre una jerarquía axiológica es también una jerarquía material (en el ordenamiento italiano, por ejemplo, no existe una jerarquía material entre una ley estatal de principio y una ley regional de detalle).

\section{Variedad de normas supremas}

La distinción entre cuatro tipos de jerarquías normativas sugiere cuatro interpretaciones distintas de la noción de norma suprema.

\subsection{Norma suprema y jerarquías formales}

En primer lugar, la norma suprema puede ser identificada con la norma que confiere el poder normativo más alto.

Si circunscribirnos el discurso al horizonte de las normas positivas, y por ello mismo de los poderes constituidos, en los ordenamientos con una constitución rígida el poder normativo más alto es el poder de revisión constitucional; en los ordenamientos con una constitución flexible es el poder legislativo. La norma fundamental suprema será en consecuencia, según sea el caso, aquella que confiera el poder de revisión constitucional o aquella que confiera el poder legislativo.

Pero, después de todo, ¿por qué circunscribir el discurso a los poderes constituidos? Parece natural pensar que el poder normativo más alto en el ámbito de un ordenamiento jurídico sea, no ya el poder de revisión constitucional (que es, precisamente, un poder constituido), sino el poder constituyente mismo. De modo que parece también natural pensar -como Kelsen- que norma suprema de todo ordenamiento sea no ya una norma constitucional, positiva,

${ }^{8}$ Aludo a la relación entre leyes estatales de principio y leyes regionales de detalle como se configuran en el art. 117 de la Constitución italiana (N. del T. Puede encontrarse un ejemplo paralelo en el ordenamiento español entre las leyes marco y las leyes autonómicas de desarrollo, tal como se establece en el artículo 150 de la Constitución española). 
sino una norma meta-constitucional, no-positiva, no puesta sino presupuesta: en suma, aquella norma «fundamental» que confiere poder normativo a los autores de la constitución (o a los autores de la «primera» constitución histórica, en el caso de una secuencia temporal de constituciones, cada una de las cuales sea autorizada por la constitución precedente) ${ }^{9}$.

\subsubsection{Dos errores de Kelsen}

Creo que la tesis de Kelsen nace de la conjunción de dos errores.

El primer error es la confusión -muy conocida- entre validez y existencia (o pertenencia), en virtud de la cual Kelsen no puede admitir que en un ordenamiento existan normas no-válidas, y en particular normas «soberanas» o «independientes», esto es, ni válidas ni inválidas. Si validez es existencia, entonces no puede efectivamente existir una norma no-válida, es decir, no-existente.

El segundo error es la confusión entre criterios de validez y/o existencia y normas que confieren poderes.

Así:

(1) En primer lugar, validez y existencia (o pertenencia) son nociones distintas ${ }^{10}$. (En el uso común, si no me equivoco, el vocablo "validez" es usado para designar lo que yo, aquí, llamo "existencia").

(1.1.) Grosso modo (dejando de lado, por razones de simplicidad, los criterios de validez de las normas implícitas, a las que anteriormente he hecho referencia), en los ordenamientos contemporáneos, o en gran parte de ellos, se llama válida a toda norma que:

(a) haya sido producida formalmente de acuerdo a las normas sobre la producción jurídica -o meta-normas- que regulan su producción (validez formal), y

${ }^{9}$ H. Kelsen, Introducción to the Problems of Legal Theory, cit., págs. 59 y ss.; Id., General Theory of Law and State, cit., págs. 115 y ss.; Id., Théorie pur du droit, cit., 1962, págs. 261 y ss.; Id., General Theory of Norms (1979), trad. M. Hartney, Clarendon Press, Oxford, 1991, págs. 252 y ss.

${ }^{10}$ R. Guastini, Il giudice e la legge, cit., cap. V; Id., «Invalidità (atti norrnativi)», en A. Belvedere, R. Guastini, P. Zatti, V. Zeno Zencovich, Glossario, Tratado de derecho privado editado por G. Iudica y P. Zatti, Giuffrè, Milano, 1994, págs. 2356 y ss. 
(b) no sea materialmente disconforme a las normas jerárquicamente superiores que limitan su posible contenido (validez material).

(1.2.) En cambio, se predica pertenencia a un ordenamiento, o existencia, de toda norma que:

(a) incluso en ausencia de validez material, esté, al menos prima facie, provista de validez formal (haya sido producida por una autoridad competente de acuerdo al menos con algunas de las normas procedimentales que regulan la producción normativa), o bien

(b) incluso en ausencia de validez formal, haya adquirido vigencia, esto es, sea generalmente aceptada y observada por sus destinatarios (principio de efectividad) ${ }^{11}$.

En consecuencia, no toda norma perteneciente a un ordenamiento es también válida en él. No son válidas, por ejemplo, las leyes inconstitucionales (que también pertenecen al ordenamiento hasta que no sean expulsadas por el órgano competente para ello). No son válidas, sobre todo, las normas que nacen de las fuentes llamadas extra ordinem, como por ejemplo una «primera» constitución instaurada por vía revolucionaria. Con una diferencia importante: las leyes inconstitucionales son inválidas, mientras que las normas de la «primera» constitución no son ni válidas ni inválidas.

(2) En segundo lugar, los criterios de validez y/o de pertenencia -como Bulygin ha mostrado persuasivamente- no son nada más que reglas conceptuales, esto es, definiciones ${ }^{12}$. Un criterio de validez no es más que una definición de "validez" (o de "norma válida"); un criterio de existencia o pertenencia no es más que una definición de "existencia" (o de "norma existente").

Ahora bien, en el definiens de esas definiciones se mencionan normas de competencia o normas atributivas de poderes normativos. Sin embargo, una cosa es conferir un poder, es decir, dictar una norma de competencia, y otra mencionar una norma de competencia. Las normas que confieren poderes normativos son mencionadas en los criterios de validez y de pertenencia de un ordenamiento, pero no son ellas mismas criterios de validez o de pertenencia.

\subsection{Norma suprema y jerarquías materiales}

En segundo lugar, la norma suprema puede ser identificada como

${ }^{11}$ R. Guastini, Le fonti del diritto e l'interpretazione, cit., págs. 256 y ss.

${ }^{12}$ E. Bulygin, Norme, validità, sistemi normativi, cit., págs. 19 y ss., 213 y ss., 235 y ss. 
la norma de rango más elevado en la jerarquía material del ordenamiento, es decir, con aquella norma a la que ninguna otra norma puede válidamente contradecir.

Desde este punto de vista, sin embargo, no parece que haya en los ordenamientos jurídicos una sola norma suprema. Hay, más bien, una fuente suprema: la constitución en los ordenamientos con una constitución rígida, la ley en los ordenamientos con una constitución flexible.

Las normas de la constitución (o de la «primera» constitución, si se prefiere) son normas existentes en el ordenamiento, pertenecientes al mismo, y sin embargo no-válidas: más precisamente, ni válidas ni inválidas, es decir, «soberanas», «independientes» ${ }^{13}$. Ello es así por la simple razón de que en ningún ordenamiento hay normas sobre la producción constitucional (sobre la instauración de una nueva constitución) o normas superiores a la constitución, con cuya comparación pueda ser juzgada la validez o invalidez (formal o material) de la constitución ${ }^{14}$.

\subsection{Norma suprema y jerarquías lógicas}

En tercer lugar, la norma suprema puede ser identificada con la norma que se coloca en el nivel del lenguaje más alto respecto de todas las demás normas del ordenamiento.

Desde este punto de vista, la norma suprema parece ser aquella -meta-constitucional- que fija los criterios de existencia de todas las demás normas, esto es, la «regla última de reconocimiento». Como se ha mencionado ya, la «norma» en cuestión es, propiamente, una regla conceptual, es decir, una definición.

Debe observarse que la norma lógicamente suprema es aquella que establece los criterios de existencia, no de validez, de las normas. Los criterios de validez, en efecto, son normas (en sentido genérico) existentes, identificables mediante el uso de la regla de reconocimiento y, por lo tanto, no son en absoluto «supremas».

13 R. Caracciolo, El sistema jurídico. Problemas actuales, Centro de Estudios Constitucionales, Madrid, 1988, págs. 31 y ss.; E. Bulygin, Norme, validità, sistemi normativi, cit., págs. 231 y ss.; J. J. Moreso, P. E. Navarro, Orden jurídico y sistema jurídico. Una investigación sobre la identidad y la dinámica de los sistemas jurídicos, Centro de Estudios Constitucionales, Madrid, 1993, págs. 77 y ss.

${ }^{14}$ R. Guatini, Dalle fonti alle norme, II ed., Giappichelli, Torino, 1992, págs. 274 y ss.; Id., «Sur la validité de la constitution du point de vue du positivisme juridique», en M. Troper, L. Jaume (eds.), 1789 et l'invention de la constitution, Bruylant-L. G. DJ., Bruxelles-París, 1994, págs. 222 y ss. 


\subsubsection{Definiciones vs. normas de conducta}

Comúnmente, se sostiene (injustamente) que la norma lógicamente suprema de todo ordenamiento es no ya el criterio de existencia, sino el criterio de validez. Eso depende probablemente del hecho, al cual ya he hecho mención, que el término «validez» parece generalmente usado para referirse a aquello que yo llamo «existencia». Pero, en este contexto, la cosa tiene poca importancia; importa ahora advertir que los criterios (sean de validez o de existencia) no son reglas de conducta, sino definiciones. Sin embargo, por razones que no comprendo, la definición de «norma válida» (o de «norma existente») es a menudo confundida con una meta-norma iterada que obliga a obedecer a las normas válidas $^{15}$. La meta-norma en cuestión es, obviamente, una regla de conducta, aunque absolutamente vacua, ya que no prescribe otra conducta que aquella ya prescrita por las normas válidas.

No obstante, parece difícil no convenir que la definición de «norma válida» y la meta-norma que califica como obligatorias las normas válidas sean cosas conceptualmente distintas. En particular, no veo cómo se puede negar que las definiciones no son normas de conducta $^{16}$.

Por un lado, si se quiere sostener que las normas válidas son, en cuanto tales, también obligatorias o vinculantes, es necesario postular, junto a la definición de «norma válida», la existencia de una verdadera y propia norma -más exactamente, de una meta-norma- ulterior; una norma que, precisamente, itera las normas válidas, calificándolas de obligatorias, esto es, exigiendo obediencia a las mismas. Lo que no puede ser realizado por una simple definición.

Por otro lado, si se quiere sostener que existe una norma que impone la obediencia a las normas válidas, no se puede no postular contextualmente la existencia de una definición de «norma válida», sin la cual las normas a las que se debe obediencia no podrían ni siquiera ser identificadas.

${ }^{15}$ Cfr. U. Scarpelli, Cos'è il positivismo giuridico, Comunità, Milano, 1965, cap. VII; J. Raz, The Authority of Law. Essays on Law and Morality, Clarendon, Oxford, 1979, 93; N. MacCormick, H. L. A. Hart, Arnold, London, 1981, 109; J. Ruiz Manero, Jurisdicción y normas. Dos Estudios sobre función jurisdiccional y teoría del derecho, Centro de estudios constitucionales, Madrid, 1990, 142.

${ }^{16}$ C. E. Alchourrón, E. Bulygin, Análisis lógico y derecho, Centro de Estudios Constitucionales, Madrid, 1991, 439 y ss.; E. Bulygin, Norme, validità, sistemi normativi, cit., págs. 244 y ss. 


\subsubsection{Validez vs. obligatoriedad}

La infausta confusión entre el criterio de validez (o de existencia) y la meta-norma iterada (que obliga a obedecer a, predica la obligatoriedad de, las normas válidas) hace imposible distinguir entre validez y obligatoriedad. La falta de distinción entre validez y obligatoriedad, a su vez, figura en la raíz de aquella concepción -«cuasi-positivista»o falsamente positivista-, según la cual no sería posible describir un ordenamiento positivo sin «aceptar» su norma suprema ${ }^{17}$.

En cambio, la cuidada distinción entre el criterio de validez y la meta-norma iterada permite distinguir, con igual cuidado, entre validez y obligatoriedad ${ }^{18}$.

Los juicios de obligatoriedad se fundan, ciertamente, en la aceptación de la meta-norma iterada que califica como obligatorias a las normas válidas, es decir, prescribe obediencia a las mismas. Pero los juicios de validez se fundan exclusivamente en el uso del criterio de validez: no requieren en absoluto la aceptación de la meta-norma iterada. Por otro lado, el uso del criterio de validez no presupone en ningún modo su aceptación ético-política (admitido que tenga sentido hablar de aceptación ético-política en referencia a una definición, de lo que se puede dudar lícitamente), sino únicamente su conocimiento. El uso de una definición es un comportamiento meramente cognitivo, o teórico, que no implica ningún tipo de moral commitment ${ }^{19}$.

${ }^{17}$ A. Ross, «Il concetto di validità e il conflitto tra positivismo giuridico e giusnaturalismo» (1962) en A. Ross, Critica del diritto e analisi del linguaggio, ed. por A. Febbrajo y R. Guastini, II Mulino, Bologna, 1982, págs. 148 y ss.

${ }^{18}$ E. Bulygin, Norme, validità, sistemi normativi, cit., págs. 11, 65-66, 200-205.

${ }_{19}$ En el ordenamiento italiano vigente, no existe ningún criterio general de validez expresamente formulado (en realidad, los propios vocablos «válido» e «inválido» son de uso bastante raro en el lenguaje de las fuentes). Existen sin embargo, algunas disposiciones que prohíben a determinadas fuentes establecer regulaciones contrarias a otras, o confieren a determinados órganos jurisdiccionales el poder de inaplicar y/o de anular toda norma que no responda a ciertos requisitos.

Por ejemplo, el artículo 138 de la constitución italiana dispone (dicho sea de paso, en modo absolutamente oblicuo) que la ley ordinaria no puede contener normas contrarias a la Constitución-, los artículos 134 y 136 const. y el artículo 30 de la ley 87/1953 confieren a la Corte Constitucional el poder de declarar la $<$ ilegitimidad constitucional $>$ de las leyes y de los actos normativos con fuerza de ley, es decir, de anular las normas legales que sean contrarias a la Constitución; el artículo 4, I c., de las disposiciones preliminares del Código Civil italiano establece que $<$ los reglamentos no pueden contener normas contrarias a las disposiciones de las leyes>; el artículo 26 r. d. 1054/1924 y el artículo 2 de la ley 1034/1971 confieren, respectivamente, al Consejo de Estado y a los Tribunales administrativos regionales el poder anular los actos administrativos (incluidos los reglamentos) adoptados en <violación de ley>; el artículo 5 de la ley 2248/1865, ALL. E, confiere a los jueces ordinarios el 


\subsubsection{Punto de vista normativo y punto de vista cognitivo}

Es una idea difundida, a partir de Hart, que los juicios de validez son «enunciados internos»; enunciados, esto es, que presuponen la aceptación de la norma suprema del ordenamiento $^{20}$. Se llama «punto de vista interno» al ángulo visual de aquellos que aceptan esa norma suprema.

Así, si se concibe la norma suprema como una norma de conducta, es natural que el «punto de vista interno» se configure como una actitud normativa (la aceptación de una norma de conducta, precisamente). Pero si se conviene que en la noción corriente de norma suprema se confunden una regla de conducta y una definición, se obtiene que la noción de «punto de vista interno» es una noción anfibia. Una cosa es asumir un punto de vista «interno»-si se puede decir así- respecto de una definición y otra asumir un punto de vista «interno» respecto de una norma de conducta.

Debemos entonces distinguir:

(a) aquel punto de vista que supone simplemente el uso del criterio (esto es, de la definición) de validez; y

poder de inaplicar los actos administrativos (incluidos los reglamentos) que no sean $<$ conformes a las leyes $>$, etc.

De estas (y de otras) disposiciones positivas, los juristas infieren-no sin un complejo trabajo de «construcción jurídica»- los criterios de invalidez de las normas legales, de las normas reglamentarias, etc. A partir de estos criterios (particulares) de invalidez, los teóricos del derecho construyen a su vez un criterio (general) de validez. Se trata de un macro-criterio, que consiste en la conjunción de una pluralidad de distintos criterios de validez que valen para las diversas fuentes del derecho (cada uno de los cuales, por otro lado, es frecuentemente un criterio de validez meramente implícito, obtenido a contrario de un criterio expreso de invalidez).

Ni siquiera existe, por lo que conozco, en el ordenamiento italiano vigente, una meta-norma que prescriba en general la obediencia a las normas válidas.

No obstante, el artículo 54, I c., const. establece que $<$ todos los ciudadanos tienen el deber de ser fieles a la República y de observar su Constitución y sus leyes $>$; el artículo 101, II c., const. dispone a su vez que $<$ los jueces están sujetos únicamente a las leyes $>$; por otro lado, las leyes son promulgadas con la siguiente fórmula: $<_{i}$ Se obliga a cualquiera que competa a observarla [la presente ley] y hacerla observar como ley del Estado?> (artículo 1, I c., d. p. r. 1092/1985), etc.

De estas (y de otras) disposiciones, los juristas (¿o más bien: los teóricos del derecho?) obtienen la macro-norma iterada según la cual las normas válidas deben ser obedecidas por los ciudadanos y por los órganos del Estado.

${ }^{20}$ Cfr., por ejemplo, U. Scarpelli, Cos'è il positivismo giuridico, cit., cap. VII, Id., L'etica senza verità, Il Mulino, Bologna, 1982, págs. 316 ss. Cfr. G. Tarello, Diritto, enunciati, usi. Studi de teoria e metateoria del diritto, Il Mulino, Bologna, 1974, págs. 101 y ss.; A. Ross, «Il concetto di diritto secondo Hart» (1962), en S. Castignone, R. Guastini (eds.), Realismo giuridico e analisi del linguaggio. Testi di Karl Olivecrona e Alf Ross, II ed., ECIG, Genova, 1990, 315 ss.; E. Bulygin, Norme, validità, sistemi normativi, cit., págs. 51 y ss., 98 y ss. 
(b) aquel punto de vista que supone la aceptación ético-política de la meta-norma que obliga a obedecer a las normas válidas.

Uno es un punto de vista «cognitivo». El otro es un punto de vista «normativo» ${ }^{21}$. Los juicios de obligatoriedad, ya que se fundan en una norma, tienen carácter genuinamente normativo. Los juicios de validez, ya que se fundan en una definición, tienen un carácter de simple reconocimiento. Después de todo, se pueden «reconocer» las setas venenosas, sin por ello «aceptar» comérselas. ¿Por qué el reconocimiento (de la validez) de normas debería ser diverso del reconocimiento de un boletus satanas?

\subsection{Norma suprema y jerarquías axiológicas}

En cuarto lugar, la norma suprema puede ser identificada con la norma axiológicamente más importante de todo el ordenamiento.

En el pensamiento jurídico común, sin embargo, cada ordenamiento incluye, no una única norma suprema, en este sentido, sino más bien una serie de valores y/o principios axiológicamente supremos, los cuales confieren justificación y congruencia a todo el ordenamiento. Por ejemplo, son considerados principios supremos del ordenamiento italiano: la soberanía popular, la tutela de los derechos humanos, la igualdad, etc.

Tales principios son llamados supremos (o fundamentales) en el doble sentido de que fundamentan ético-políticamente a las restantes normas del ordenamiento y no necesitan a su vez de fundamento o justificación (quizás porque, a los ojos del que habla, son autoevidentes, o quizás porque son generalmente aceptados en el seno de la comunidad política de que se trate).

\subsubsection{La pretendida inmodificabilidad de los principios supremos}

El conjunto de esos principios supremos es llamado por algunos «constitución (en sentido) material». De los principios en cuestión, normalmente, algunos están meramente implícitos en el ordenamiento, mientras que otros están expresamente formulados en el texto constitucional. Estos últimos -se sostiene- no son susceptibles de revisión constitucional ${ }^{22}$.

${ }^{21}$ Algunas sugerencias en N. MacCormick, H. L A. Hart, cit., 1981, págs. 33 y ss.; J. Raz, "The Purity of the Pure Theory», en R. Tur, W. Twining (eds.), Essays on Kelsen, Clarendon, Oxford, 1986, págs. 79 y ss.; R. Vernengo, «Kelsen’s Rechtssätze as Detached Statements», ivi, págs. 99 y ss.

${ }^{22}$ Léase por ejemplo el siguiente fragmento, extraído de la sentencia 1146/1988 
Esta tesis descansa sobre un argumento que puede ser reconstruido como sigue ${ }^{23}$.

La identidad ética-política de toda constitución yace en el conjunto de los principios supremos que la caracterizan. De modo que alterar los principios en cuestión no sería distinto de la instauración de una nueva y distinta constitución. La revisión constitucional no puede, por lo tanto, avanzar hasta modificar los principios supremos de la constitución sin convertirse, por ello mismo, en una instauración constitucional.

Como es evidente, este argumento descansa a su vez sobre dos asunciones relacionadas entre sí.

La primera asunción es que una constitución no puede ser definida extensionalmente (esto es, por simple enumeración de las normas que la componen).

La segunda asunción -que se sigue de la primera- es la distinción conceptual entre revisión de la constitución existente e instauración de un nuevo ordenamiento constitucional.

$\mathrm{Si}$, en efecto, se acoge una definición puramente extensional de la constitución, entonces cada revisión constitucional -cada modificación incluso de una sola norma de la constitución existente- produce una nueva constitución ${ }^{24}$. De modo que los conceptos de revisión y de instauración constitucional se superponen enteramente.

de la Corte Constitucional italiana: $<$ La Constitución italiana contiene algunos principios supremos que no pueden ser subvertidos o modificados en su contenido esencial ni siquiera por leyes de revisión constitucional o por otras leyes constitucionales. Tales son tanto los principios que la misma constitución prevé explícitamente como límites absolutos al poder de revisión constitucional, como la forma republicana (artículo 139 Const.), como los principios que, aunque no sean expresamente mencionados entre aquellos no sujetos al procedimiento de revisión constitucional, pertenecen a la esencia de los valores supremos sobre los que se funda la Constitución italiana. Esta Corte, por lo demás, ha ya reconocido en numerosas decisiones que los principios supremos del ordenamiento constitucional tienen un valor superior respecto de las otras normas o leyes de rango constitucional, sea cuando ha sostenido que también las disposiciones del Concordato, las cuales gozan de la particular «cobertura constitucional» provista en el artículo 7, II, Const., no se sustraen a la comprobación de su conformidad con los «principios supremos del ordenamiento constitucional» [...], sea cuando ha afirmado que la ley de ejecución del Tratado de la CEE puede ser sometida al control de esta Corte «en referencia a los principios fundamentales de nuestro ordenamiento constitucional y a los derechos inalienables de la persona humana» [...]. No se puede, por tanto, negar que esta Corte sea competente para juzgar la conformidad de las leyes de revisión constitucional y de las otras leyes constitucionales también respecto a los principios supremos del ordenamiento constitucional. Si no fuese así, por lo demás, se llegaría al absurdo de considerar el sistema de garantías jurisdiccionales de la Constitución como defectuoso o no efectivo precisamente en relación a sus normas de más elevado valor>.

${ }^{23}$ R. Guastini, «Revisione costituzionale: problemi di forma e di sostanza», en Ragion pratica, 3, 1994, págs. 245-255.

${ }^{24}$ E. Buligyn, Norme, validità, sistemi normativi, cit., págs. 76 y ss. 
Naturalmente, no hay nada malo en preferir una definición intensional de la constitución, identificando cada constitución a partir de los principios supremos que la caracterizan. El único problema es que el predicado «principio supremo» no denota una propiedad empírica, de forma que no hay otro modo de identificar un principio supremo que a través de un juicio de valor, como tal fatalmente controvertible.

(Traducción de Jordi Ferrer) 